\title{
Clinical interventions and speech outcomes for individuals with submucous cleft palate
}

\author{
Seung Eun Jung ${ }^{1}$, Seunghee $\mathrm{Ha}^{1}$, Kyung S. Koh ${ }^{2}$, Tae Suk $\mathrm{Oh}^{2}$ \\ ${ }^{1}$ Division of Speech Pathology and Audiology, Audiology and Speech Pathology Research Institute, Hallym University, Chuncheon; \\ ${ }^{2}$ Department of Plastic Surgery, Asan Medical Center, Seoul, Korea
}

\begin{abstract}
Background This study aimed to identify the initial diagnostic characteristics and treatment status of children with submucous cleft palate (SMCP) and to examine the relationship between the timing of surgical correction and the degree of articulation and resonance improvement. Methods This retrospective study included 72 children diagnosed with SMCP between 2008 and 2016. The evaluation criteria were the age of the initial visit, total number of visits, age at the end of treatment, speech problems, resonance problems, and speech therapy.

Results Children with SMCP first visited the hospital at an average age of 34.32 months, and speech problems were identified at an average age of 48.53 months. Out of 72 children, 46 underwent surgery at an average age of 49.74 months. Four of these children required secondary surgery at an average age of 83.5 months. Among the children who underwent surgery before 3 years of age, 70\% exhibited articulation improvements, with mild-to-moderate hypernasality. Articulation improvements showed no statistically significant differences according to age at the time of surgery. However, children who underwent surgery before 4 years had a better hypernasality rating than those who underwent surgery after 4 years of age.

Conclusions Children with SMCP tend to undergo delayed treatment because the anatomical symptoms in some children with SMCP are unclear, and surgical interventions are considered only after speech problems are clarified. Starting interventions as early as possible reduces the likelihood of receiving secondary surgery and speech therapy, while increasing expectations for positive speech function at the end.
\end{abstract}

Keywords Submucous cleft palate / Velopharyngeal insufficiency / Clinical study / Surgical procedure / Speech disorders

\author{
Correspondence: Seunghee Ha \\ Division of Speech Pathology and \\ Audiology, Audiology and Speech \\ Pathology Research Institute, Hallym \\ University, 1 Hallimdaehak-gil, \\ Chuncheon 24252, Korea \\ Tel: +82-33-248-2215 \\ Fax: +82-33-256-3420 \\ E-mail: shha@hallym.ac.kr \\ Kyung S. Koh \\ Department of Plastic Surgery, Asan \\ Medical Center, University of Ulsan \\ College of Medicine, 88 Olympic-ro \\ 43-gil, Songpa-gu, Seoul 05505, \\ Korea \\ Tel: +82-2-3010-3603 \\ Fax: $+82-2-476-7471$ \\ E-mail: kskoh@amc.seoul.kr
}

Received: April 17, $2020 \bullet$ Revised: August 19, $2020 \bullet$ Accepted: August 20, 2020

pISSN: 2234-6163 • elSSN: 2234-6171 • https://doi.org/10.5999/aps.2020.00612 • Arch Plast Surg 2020;47:542-550

\section{INTRODUCTION}

Submucous cleft palate (SMCP) refers to an innate defect at the back of the palate with no damage of the mucous membrane. Calnan [1] proposed that the presence of a bifid uvula, palatal muscle diastasis, and a notch in the posterior surface of the hard palate are three characteristic symptoms of SMCP. The observa- tion of all three characteristics during a physical exam, representing a clear classic triad, is termed overt or classic SMCP. Defects can also manifest as occult SMCP, which does not show clear anatomical symptoms, but instead presents with abnormal symptoms such as velopharyngeal insufficiency (VPI). Additionally, the extent of anatomical and functional defects substantially varies in SMCP. 
The reported incidence of SMCP varies according to the diagnostic criteria [2-4]. Weatherley-White et al. [5] reported SMCP in nine out of 10,836 children $(0.08 \%)$, whereas Garcia Velasco et al. [6] reported only one SMCP case among 6,000 children $(0.02 \%)$. However, these two studies reported different incidence rates of SMCP based on anatomical defects and excluded occult SMCP, which does not show clear symptoms except for hypernasality. In a study that included children with occult SMCP, ten Dam et al. [2] reported SMCP in 28 out of 800 children (3.5\%). Gosain et al. [7] suggested that because the incidence of bifid uvula in previous studies ranged from $1 \%$ to $7.5 \%$, the incidence of occult SMCP may be higher than that has been reported at present.

Children with SMCP are often diagnosed at a late stage when a child articulates incorrectly or shows hypernasality. The average age of children diagnosed with SMCP was reported to be 4.9 years by Reiter et al. [8] and 3.6 years by $\mathrm{Ha}$ et al. [9]. Oji et al. [10] reported the presence of bifid uvula in children with overt SMCP who had been diagnosed at an average age of 19.5 months. In the study conducted by Oji et al. [10], children with occult SMCP presented only with speech problems, without any anatomical symptoms, and were diagnosed at an average age of 56.8 months.

The reported incidence of hypernasality in children with $\mathrm{SMCP}$ is $5 \%$ to $50 \%$. In these patients, surgical intervention is considered to be the optimal treatment. Because the timing of surgery is generally considered to be an important factor that determines speech outcomes, children who received early surgical intervention had better outcomes [11-14].

Several studies have suggested that a younger age leads to better results of surgery in children with SMCP, but research on the different effects of treatment depending on the timing of surgery remains insufficient. Pensler and Bauer [15] reported that six of eight children who underwent surgery before the age of 2 years had normal speech outcomes, as opposed to only one in seven children who underwent surgery after the age of 2 years. Abyholm [16] compared the speech outcomes in a group of 7 -year-old children who underwent surgery and reported that $84 \%$ of children who underwent surgery before the age of 7 years showed normal nasality or minimal-to-mild hypernasality, whereas only $64 \%$ of children who underwent surgery after the age of 7 years showed the same results. Ha et al. [9] conducted a retrospective study to determine the optimum age to treat 92 children with SMCP. They reported that those who received surgical intervention before the age of 18 months had a lower probability of undergoing secondary surgery and having speech errors. Moreover, Baek et al. [17] reported that hypernasality was highly likely to persist in children who received surgical in- tervention after the age of 5.5 years. Ettinger et al. [18] reported no significant difference in postoperative hypernasality scores between an early surgery group ( $2.42 \pm 0.88$ years $)$ and a late surgery group ( $6.41 \pm 1.93$ years).

The optimal intervention strategies for cleft lip and palate, as well as for SMCP, taking into account the appropriate age, stages, and methods of intervention, remain controversial. Owing to the diverse anatomical characteristics of SMCP, the spectrum of clinical interventions is considerably broad. Each child may require a different treatment process, and it is thus difficult to combine different intervention processes into a single strategy. Because treatments directly affect outcomes, it is essential to systematically identify an optimal treatment process by investigating the procedures involved, differences among the treatment processes, and other influential factors [19]. The purpose of this study was to investigate the initial diagnostic characteristics and treatment status of individuals with SMCP and to examine the relationship between the timing of surgical correction and the degree of articulation and resonance improvement.

\section{METHODS}

\section{Subjects}

The study retrospectively analyzed data for 117 children diagnosed with SMCP out of 700 individuals diagnosed with cleft palate from 2008 to 2016 at the Asan Medical Center Cleft Palate Clinic in South Korea. The study was approved by the Institutional Review Board of Asan Medical Center (IRB No. 20141040) and performed in accordance with the principles of the Declaration of Helsinki. Twenty-four children with cleft lip, as well as 14 children who had medical records of hospital visits but lacked speech evaluation results, were excluded from this study. Furthermore, seven children in whom a speech evaluation was impossible owing to intellectual disability or serious speech-language disorders were excluded from the analysis. Consequently, 72 children were included in this study. The characteristics of the patients with SMCP are shown in Table 1. Twelve children had comorbid conditions, including seven with velocardiofacial syndrome.

Among the subjects, 16 children had normal hearing at the time of their first visit to the clinic, whereas 49 children were diagnosed with frequent otitis media. Patients with congenital hearing loss or other problems unrelated to otitis media were excluded from this study. No records of hearing problems were found in 29 children. All patients underwent Furlow double-opposing $\mathrm{Z}$ palatoplasty. The subjects were divided into four groups according to their age at the time of surgery: $<3$ years, between 3 and 4 years, $>4$ years, and no surgery. A total of 22 
Table 1. Characteristics of patients with submucous cleft palate by group

\begin{tabular}{|c|c|c|c|c|c|}
\hline \multirow{2}{*}{ Variable } & \multirow{2}{*}{ All patients } & \multicolumn{3}{|c|}{ Age at surgery, No. (\%) } & \multirow{2}{*}{ Unoperated group } \\
\hline & & $<3 \mathrm{yr}$ & $3-4 \mathrm{yr}$ & $>4 \mathrm{yr}$ & \\
\hline Sex & 72 & 16 & 11 & 19 & 26 \\
\hline Male & $38(52.8)$ & $10(62.5)$ & $4(36.4)$ & $13(68.4)$ & $11(42.3)$ \\
\hline Female & $34(47.2)$ & $6(37.5)$ & $7(63.6)$ & 6 (31.6) & $15(57.7)$ \\
\hline Syndrome & 12 & 1 & 3 & 4 & 4 \\
\hline Velocardiofacial syndrome & $7(58.3)$ & 0 & $2(66.7)$ & $3(75.0)$ & $2(50.0)$ \\
\hline Others & $5(41.7)$ & $1(100)$ & $1(33.3)$ & $1(25.0)$ & $2(50.0)$ \\
\hline \multicolumn{6}{|l|}{ Hearing problems } \\
\hline No loss & $15(20.8)$ & $4(25.0)$ & $4(36.4)$ & $3(15.8)$ & $4(15.4)$ \\
\hline Problems & 38 (52.8) & $11(68.8)$ & $6(54.5)$ & $14(73.7)$ & 7 (26.9) \\
\hline Unknown & $19(26.4)$ & $1(6.3)$ & $1(9.1)$ & $2(10.5)$ & $15(57.7)$ \\
\hline
\end{tabular}

children underwent surgery before the age of 3 years, 17 underwent surgery between the ages of 3 and 4 years, and 20 underwent surgery after the age of 4 years; however, 35 children did not receive any surgical intervention.

\section{Speech assessment procedures}

The data on sex, age, palatal surgery, and speech assessment and therapy were obtained from the subjects' electronic medical records. Preoperative and postoperative assessments included intraoral and perceptual speech examinations. Perceptual speech assessments consisted of articulation errors, resonance functions, nasal emissions, voice, and intelligibility.

The Asan Medical Center Clinic proceeds with medical treatment and speech assessment when a child with suspected cleft palate visits the hospital. Clinical decisions are usually postponed until the child is able to produce connected speech comprising sentences with four or five words. Comprehensive speech evaluations were performed to determine the overall medical procedures, including the feasibility of surgery and speech therapy.

Speech evaluations were performed by a single speech-language pathologist. During evaluations, articulation was judged based on compensatory errors, developmental errors, and obligatory errors $(0=$ no problem, $1=$ has problem $)$, and the overall severity of articulation problems was determined by aggregating the results. Additionally, hypernasality was evaluated by classifying the subjects into seven different grades $(0=$ normal, $1=$ minimal, $2=$ mild, $3=$ mild to moderate, $4=$ moderate, $5=$ moderate to severe, $6=$ severe) using a 2-point nasal emission scale (has problem versus no problem). Language and voice issues were assessed based on parental reports and screening tests (has problem vs. no problem). If articulation problems were revealed through speech evaluation, speech therapy was recommended or performed. However, when VPI was apparent and it was judged that this problem had an obvious negative effect on speech prob- lems, surgery could be performed before speech therapy.

Speech assessments were performed at 6-month to 1-year intervals from the moment the children could undergo speech assessment until the patient was discharged from treatment. In cases where in the children had not yet fully achieved speech output and could not undergo speech assessment, parental education was provided to promote language development in those children. When surgery for the child was decided upon, preoperative assessments along with postoperative assessment at 1-3 months after surgery were conducted. Furthermore, improvements in articulation and resonance after surgery were analyzed by performing a speech assessment after treatment $(0=$ no improvement, $1=$ improvement, 2 = originally normal, $3=$ missing preoperative or postoperative assessment; no data for comparison).

The choice was made to discharge the patient from treatment and evaluations when speech and resonance were maintained at normal or mild levels and further intervention was considered unnecessary by the doctor, caregivers, and children. All children whose chart records showed normal speech and resonance grades were deemed to have been discharged normally.

\section{Statistical analysis}

Descriptive statistical analyses were performed to investigate the initial diagnostic characteristics and treatment status of individuals, and the chi-square test was conducted to examine the relationship between the timing of surgical correction and the degree of articulation and resonance improvement. SPSS version 25.0 (IBM Corp., Armonk, NY, USA) was employed to conduct the statistical analysis.

\section{RESULTS}

\section{Clinical status after initial diagnosis}

The age at initial diagnosis is shown for the entire group in Table 2. The initial visit occurred at an average age of 34.32 months 
Table 2. Characteristics of patients at the initial diagnosis

\begin{tabular}{|lccccc|}
\hline \multirow{2}{*}{ Variable } & All & \multicolumn{3}{c}{ Age at surgery } & Unoperated \\
\cline { 3 - 5 } & patients & $<\mathbf{~ y r}$ & $\mathbf{3 - 4} \mathbf{~ y r}$ & $\mathbf{>} \mathbf{~ y r}$ & group \\
\hline No. of patients & 72 & 16 & 11 & 19 & 26 \\
Age at first visit (mon) & & & & & \\
$\quad$ Mean & 34.32 & 11.31 & 33.18 & 65.05 & 26.50 \\
$\quad$ Range & $0-144$ & $1-32$ & $13-44$ & $3-144$ & $0-108$ \\
Age at initial speech & & & & & \\
$\quad$ assessment (mon) & & & & & \\
$\quad$ Mean & 48.53 & 32.69 & 40.09 & 72.89 & 44.04 \\
Range & $22-145$ & $23-45$ & $34-45$ & $31-145$ & $22-108$ \\
\hline
\end{tabular}

Table 3. Clinical status following the initial diagnosis

\begin{tabular}{|c|c|c|c|c|c|}
\hline \multirow{2}{*}{ Variable } & \multirow{2}{*}{$\begin{array}{l}\text { Total } \\
\text { group }\end{array}$} & \multicolumn{3}{|c|}{ Age at surgery } & \multirow{2}{*}{$\begin{array}{l}\text { Unoperated } \\
\text { group }\end{array}$} \\
\hline & & $<3 \mathrm{yr}$ & $3-4 \mathrm{yr}$ & $>4 \mathrm{yr}$ & \\
\hline No. of patients & 72 & 16 & 11 & 19 & 26 \\
\hline \multicolumn{6}{|l|}{$\begin{array}{l}\text { No. of routine follow-up } \\
\text { assessments (times) }\end{array}$} \\
\hline Mean & 6.53 & 8.38 & 7.00 & 7.68 & 4.35 \\
\hline Range & $1-16$ & $5-15$ & $4-13$ & $4-16$ & $1-12$ \\
\hline \multicolumn{6}{|l|}{$\begin{array}{l}\text { Age at final speech } \\
\text { assessment (mon) }\end{array}$} \\
\hline Mean & 65.9 & 54.25 & 54.91 & 94.95 & 56.5 \\
\hline Range & 24-162 & 24-105 & $38-90$ & 50-162 & $33-133$ \\
\hline
\end{tabular}

(range, 0-144 months) and the average age at which the speech problem was first identified through speech evaluation was 48.53 months (range, $22-145$ months).

At the time of initial visit, the average age of children who underwent surgery before 3 years, between 3 and 4 years, and after 4 years was 11.31 months (range, 1-32), 33.18 months (range, 13-44), and 65.05 months (range, 3-144), respectively; however, the average age of children who did not undergo surgery was 26.5 months (range, 0-108 months). At the age of 32.69-72.89 months, speech problems were diagnosed via detailed speech evaluations.

The characteristics of the children's visits are shown in Table 3. From the initial visit to the end of treatment, the average number of times that the children visited the hospital was 6.53 months (range, 1-16 months), and the average age of the children at their final visit was 65.9 months (range, 24-162 months).

The average visitation frequency in the surgical intervention groups ranged between 7 and 8.38 times, and that for the nonsurgical group was 4.35 times. During their last visit, the average ages at which the children aged $<3$ years, between 3 and 4 years, and $>4$ years underwent surgery were 54.25 months (range, 24-105), 54.91 months (range, 38-90), and 94.95 months (range, 50-162), respectively. The average of the children in the group that did not receive surgery was 56.5 months

\section{Table 4. Surgical characteristics}

\begin{tabular}{|lcccc|}
\hline \multirow{2}{*}{ Variable } & \multirow{2}{*}{$\begin{array}{c}\text { All } \\
\text { patients }\end{array}$} & \multicolumn{4}{c|}{ Age at surgery } \\
\cline { 3 - 6 } & & $<\mathbf{~ y r}$ & $\mathbf{3 - 4} \mathbf{~ y r}$ & $>4 \mathbf{~ y r}$ \\
\hline First palatal repair & & & & \\
No. of patients & 46 & 16 & 11 & 19 \\
Mean (mon) & 49.74 & 18.81 & 42.36 & 80.05 \\
Range (mon) & $11-146$ & $11-33$ & $36-47$ & $49-146$ \\
Second palatal repair & & & & \\
No. of patients & 4 & 0 & 2 & 2 \\
Mean (mon) & 83.5 & - & 69.5 & 97.5 \\
Range (mon) & $61-100$ & - & $61-78$ & $95-100$ \\
\hline
\end{tabular}

(range, 33-133 months) at the time of their last visit.

Out of 72 children, 46 underwent surgery at an average age of 49.74 months, and four children underwent secondary surgery at an average age of 83.5 months (range, 61-100 months); 16 children aged $<3$ years underwent surgery at an average age of 18.81 months (range, 11-33 months), and none of these children required additional surgery. Eleven children aged between 3 and 4 years underwent surgery at an average age of 42.36 months (range, 36-47 months), whereas 19 children underwent surgery after the age of 4 years, two of whom required secondary surgery. More detailed information is provided in Table 4.

\section{Speech outcomes of individuals with SMCP who received surgery}

The characteristics of speech problems before and after surgery for all the groups are shown in Table 5. Out of the 72 children who received surgery, 57 (79.2\%) exhibited speech problems, including compensatory articulation errors and developmental articulation errors, while 55 children (76.4\%) exhibited hypernasality above a mild level. At the end of treatment, 39 children (53.4\%) continued to experience articulation problems above a mild level, while 28 experienced resonance problems. Thirtythree children showed improvements in articulation owing to treatment and 10 children did not. Ten other children had insufficient information available regarding improvement in articulation, and the remaining 10 children showed normal findings regarding articulation problems during the initial evaluation. Forty-six children (63.9\%) showed improvements in resonance, and six children (8.3\%) did not show improvements in hypernasality. Speech therapy was recommended for 38 children at the initial evaluation, and for three children, it was recommended at a later period; a total of 11 children completed speech therapy.

To compare surgical outcomes, a statistical analysis was performed of the children who underwent surgery. Out of 72 children, 46 received Furlow palatoplasty. Four of these children lacked additional speech evaluation records after surgery and 
Table 5. Analysis of initial and final speech issues

\begin{tabular}{|c|c|c|c|c|c|c|c|c|}
\hline \multirow{3}{*}{ Speech component } & \multirow{2}{*}{\multicolumn{2}{|c|}{ Total group $(n=72)$}} & \multicolumn{6}{|c|}{ Age at surgery, No. (\%) } \\
\hline & & & \multicolumn{2}{|c|}{$<3$ yr $(n=16)$} & \multicolumn{2}{|c|}{$3-4$ yr $(n=11)$} & \multicolumn{2}{|c|}{$>4$ yr $(n=19)$} \\
\hline & Pre & Post & Pre & Post & Pre & Post & Pre & Post \\
\hline Articulatory problems & 57 (79.2) & $39(53.4)$ & $12(75.0)$ & $6(37.5)$ & $11(100)$ & $5(45.5)$ & $18(94.7)$ & $16(84.2)$ \\
\hline Compensatory errors & 27 (37.5) & 14 (19.2) & $4(25.0)$ & 0 & $11(100)$ & $5(45.5)$ & $8(42.1)$ & $6(31.6)$ \\
\hline Hypernasality & 55 (76.4) & $28(38.4)$ & $4(25.0)$ & $3(18.8)$ & $11(100)$ & $5(45.5)$ & $18(94.7)$ & $9(47.4)$ \\
\hline Nasal emission & $26(36.1)$ & $19(26.4)$ & $14(87.5)$ & $1(6.3)$ & $3(27.3)$ & $3(27.3)$ & $12(63.2)$ & $7(36.8)$ \\
\hline \multicolumn{9}{|l|}{ Speech therapy ${ }^{a)}$} \\
\hline 1 & $38(52.8)$ & $32(44.4)$ & $4(25.0)$ & $4(25.0)$ & $11(100)$ & $5(45.5)$ & $12(63.2)$ & $13(68.4)$ \\
\hline 2 & - & $11(15.3)$ & - & $3(18.8)$ & - & $3(27.3)$ & - & $2(10.5)$ \\
\hline
\end{tabular}

Pre, pre-surgery; Post, post-surgery.

a) Speech therapy: 1 = under treatment or recommended to receive treatment; 2 = discharged from speech treatment.

Table 6. Analysis of articulation improvements

\begin{tabular}{|lclr|}
\hline \multirow{2}{*}{ Group } & \multicolumn{3}{c|}{ Age at surgery, No. (\%) } \\
\cline { 2 - 4 } & $<3 \mathrm{yr}$ & $\mathbf{3 - 4} \mathbf{~ y r}$ & $>4 \mathrm{yr}$ \\
\hline Improvement & $11(73.3)$ & $7(77.8)$ & $10(55.6)$ \\
No improvement & 0 & $2(22.2)$ & $6(33.3)$ \\
Normal range at initial visit & $4(26.7)$ & 0 & $2(11.1)$ \\
Total & $15(100)$ & $9(100)$ & $18(100)$ \\
\hline
\end{tabular}

were excluded from the analysis of surgical outcomes, leaving only the records of 42 children available for analysis.

The results of the analysis of improvements in articulation in different groups are shown in Table 6. In the group that underwent surgery before the age of 3 years, 11 children (73.3\%) showed improvements in articulation. The remaining four children $(26.7 \%)$ in this group had normal articulation when they first visited the clinic. In the group that underwent surgery between the ages of 3 and 4 years, seven children (77.8\%) showed improvements in articulation and two (22.2\%) did not; none of the children in this group remained at a normal level from the time of the initial evaluation. Ten children $(55.6 \%)$ who received surgery after the age of 4 years showed improvements in articulation and six (33.3\%) did not. Two (11.1\%) of the children in this group remained at a normal level from the time of the initial evaluation. The chi-square test showed no significant difference in articulation improvements depending on the timing of the operation $\left(\chi^{2}=8.456, \mathrm{P}>0.05\right)$.

The hypernasality grades before and after treatment for the children who underwent surgery are shown in Fig. 1. The average hypernasality grades were 3.28 points and 2.22 points before and after surgical treatment, respectively. The first speech evaluation for the group that underwent surgery before the age of 3 years was mainly conducted after surgery. Therefore, these results were excluded from the analysis. The final hypernasality
Fig. 1. Hypernasality grades before and after treatment

a) Hypernasality grade: $0=$ normal, $1=$ minimal, $2=$ mild, $3=$ mild to moderate, $4=$ moderate $5=$ moderate to severe, $6=$ severe.

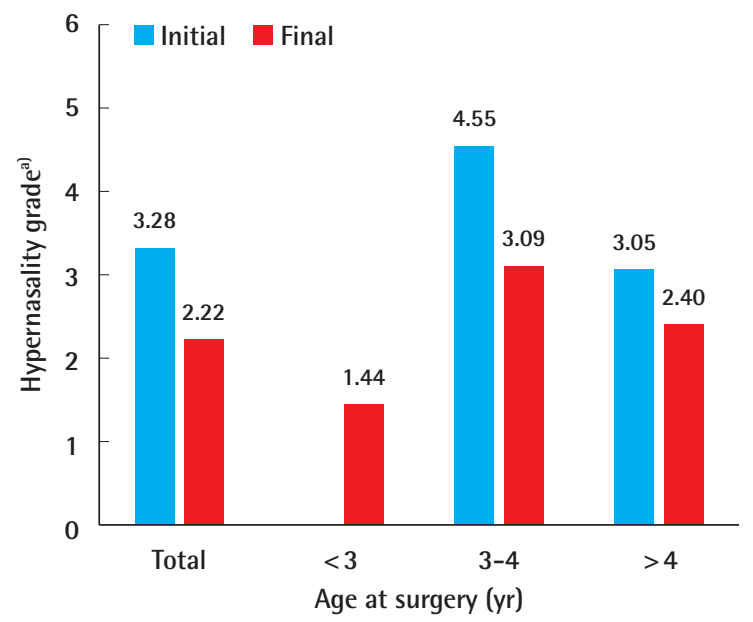

grade for this group was 1.44 points. The group that underwent surgery between the ages of 3 and 4 years showed a change in hypernasality from 4.55 points at the time of the initial visit to 3.09 points at the end of treatment, whereas the group that underwent surgery after the age of 4 years showed a change from 3.05 to 2.40 points.

The analysis of hypernasality grade at the final assessment is presented in Table 7. None of the children in the group that underwent surgery before the age of 3 years showed hypernasality above a moderate level at their final visit. Thirteen children (86.6\%) had normal levels of nasality; additionally, one child (6.7\%) had a mild level of hypernasality and another child (6.7\%) had a mild-to-moderate level. With regard to hypernasality, in the group that underwent surgery between the ages of 3 and 4 years, five children (55.6\%) had a normal level of nasali- 
Table 7. Analysis of hypernasality grade at the final assessment

\begin{tabular}{|lccc|}
\hline \multirow{2}{*}{ Group } & \multicolumn{3}{c|}{ Age at surgery, No. (\%) } \\
\cline { 2 - 4 } & $<\mathbf{~ y r}$ & $\mathbf{3 - 4} \mathbf{~ y r}$ & $>\mathbf{4 ~ y r}$ \\
\hline Normal & $13(86.6)$ & $5(55.6)$ & $5(27.8)$ \\
Minimal & 0 & $1(11.1)$ & $5(27.8)$ \\
Mild & $1(6.7)$ & 0 & $6(33.3)$ \\
Mild to moderate & $1(6.7)$ & 0 & $1(5.6)$ \\
Moderate & 0 & 0 & $1(5.6)$ \\
Moderate to severe & 0 & $1(11.1)$ & 0 \\
Severe & 0 & $2(22.2)$ & 0 \\
Total & $15(100)$ & $9(100)$ & $18(100)$ \\
\hline
\end{tabular}

ty, one child had a moderate-to-severe level of hypernasality, two children $(22.2 \%)$ had a severe level, and one (11.1\%) had a minimal level. In the group that underwent surgery after the age of 4 years, six children (33.3\%) had mild levels of hypernasality, five children $(27.8 \%)$ had normal or minimal levels, and one (5.6\%) had a mild-to-moderate level. The chi-square test showed a significant relationship between receiving a surgical intervention before the age of 3 years and the degree of hypernasality $\left(\chi^{2}=4.475, \mathrm{P}<0.001\right)$. Furthermore, a post-hoc analysis showed no difference in the degree of postoperative hypernasality between the group that underwent surgery before the age of 3 years and the group that underwent surgery between the ages of 3 and 4 years; however, a significant difference in hypernasality was noted between the group that underwent surgery before the age of 3 years and the group that underwent surgery after the age of 4 years, as well as between the group that underwent surgery between the ages of 3 and 4 years and the group that underwent surgery after the age of 4 years.

\section{DISCUSSION}

In this study, speech problems were identified at an average age of 48.53 months after administering speech tests to children with SMCP; this result is consistent with the findings from previous studies, thereby indicating that the diagnosis of SMCP is often delayed $[2,7,8,10,20]$. Possible reasons for the delay in diagnosis are as follows: (1) symptoms or signs of SMCP are unclear or mild; (2) there are some cases in which abnormal structures do not become apparent until the age of 4-5 years [8]; and (3) the incidence of SMCP in the general population is considered to be between $0.02 \%$ and $0.08 \%$, and the physicians are unfamiliar with this unusual malformation $[10,20]$.

Few studies have investigated the number of clinic visits by SMCP patients. In this study, the number of visits was not significantly correlated with the age at surgery. Clinic visits are usu- ally costly and stressful, which are some of the most important factors that negatively affect the quality of life and treatment satisfaction of patients, with consequent impacts on the treatment satisfaction rates among patients, ultimately affecting the final outcome. However, in this study, all three groups had a similar frequency of clinic visits.

The age at treatment discharge was 65.9 months on average, which is slightly earlier than the age reported in other countries [12]. These findings could be attributed to the fact that some children, who were recommended to receive follow-up for observation after the resolution of their major speech problems via surgical intervention and speech therapy, did not return for follow-up visits. An in-depth analysis of the hospital's electronic chart for this study revealed that $29.2 \%$ of the children did not continue with speech evaluation follow-ups after one visit following surgery, and $22.2 \%$ of the children whose speech problems were confirmed to be resolved did not participate in the final evaluation after having undergone 1 year of observation.

As with overt cleft palate, SMCP can be accompanied by VPI. It has generally been accepted, without any significant disagreements, that speech problems occur secondary to the aforementioned problems [21]. Speech problems associated with VPI occur in up to $80 \%$ of SMCP patients [9]. Several studies have reported that hypernasality above a mild level occurs in most SMCP patients [22,23]. In this study, the prevalence of problems with articulation and resonance at the time of the initial evaluation were similar to that reported in previous studies $[9,20]$.

Proponents of palatal reconstruction suggest that normalization of primary anatomical abnormalities is a logical first step for improving velopharyngeal function $[3,5,15,24]$. The timing and types of procedures that produce optimal outcomes still remain controversial [7], but many researchers believe that surgical intervention is the most effective way to resolve VPI in children with SMCP. A literature review by Gilleard et al. [25] revealed that between 2001 and 2012, surgery led to normal speech in $33 \%$ to $89 \%$ of patients $[2,8,24,26]$. However, all of these studies reported postoperative outcomes based on the surgical technique, but not age.

The surgical timing and appropriate surgical techniques for SMCP also remain controversial $[10,15,25,27]$. Early surgical intervention seems to be essential for avoiding improper speech habits, including compensatory articulation, and for enabling early speech interventions. However, the possible inaccuracy of speech assessments in children aged $<2$ years is assumed to be a limitation. Pensler and Bauer [15] concluded that even if children were diagnosed with SMCP before the age of 2 years owing to structural problems, intervention decisions should be made based on the presence of VPI. Many researchers have also 
suggested that early diagnosis and surgery are important factors for successful treatment $[7,10,17,26,27]$, whereas other researchers have suggested that the timing of surgery is not a predictor of speech output [24].

In this study, none of the children in the group that underwent surgery before the age of 3 years required secondary surgery; this result is consistent with the finding by a previous study [9] that early surgical intervention reduced the risk of additional surgery. All children in the group that underwent surgery before the age of 3 years showed an improvement in articulation. However, $33 \%$ of the children in the group that underwent surgery after the age of 4 years did not show an improvement in articulation, although the differences did not reach the level of statistical significance.

At the time of the final evaluation, the results suggest that the later the operation time, the longer it takes to resolve articulation problems. In particular, compensatory articulations were eliminated in all of the children who underwent surgery before the age of 3 years; this result is consistent with previously reported findings that early intervention prevents improper speech habits such as compensatory articulation [15]. Moderate and more severe postoperative problems were also the most common in the group that underwent surgery after the age of 4 years (68.4\%). These results suggest that the earlier a child undergoes surgery, the less severe the articulation problems will be.

Additionally, the earlier the children underwent surgery, the less likely they were to require speech therapy. For children with SMCP along with speech problems, it is essential to combine speech therapy with surgical intervention for achieving the most optimal speech outcomes. Thus, a significant strength of this study is that there was less need for speech therapy in patients who received early surgical intervention. However, owing to the treatment protocol at the clinic, it cannot be overlooked that children who underwent surgery at an early age visited the hospital during the period of language development, and parental education provided by the clinic may have had a positive effect on appropriate articulation in such children.

Thirty-nine (92.9\%) of the 42 patients who underwent surgery showed an improvement in postoperative hypernasality, which is consistent with the findings from a previous study [19]. There was no statistically significant difference in the improvements in hypernasality between the groups, but significant differences were found in the final hypernasality level. The results could have been significantly affected by the initial evaluation, which was performed after surgery in the group that underwent surgery before the age of 3 years. Furthermore, the small number of subjects and the uneven number of subjects in each group likely had a negative impact. Ha et al. [9] reported that the tim- ing of surgery did not significantly correlate with VPI results, but a significant correlation was observed in this study. Additionally, none of the children who underwent surgery before the age of 3 years had hypernasality above the moderate level.

While summarizing patients' electronic charts for this study, we were able to confirm that children's conditions constantly changed throughout the treatment process. Because these decisions are not all made based on a single initial diagnosis, regular and consistent observation and treatment are considered to be important for SMCP, similar to cleft palate. Additionally, we acknowledge that the intervention of speech therapy should be analyzed in a complex manner. Although all groups were administered speech therapy under the same standard conditions, the group that received surgical intervention after the age of 4 years may have contained children who did not receive adequate speech therapy owing to a delay in their first hospital visit. We acknowledge that this may have affected the children's final speech function.

Because this study was conducted among patients treated with the same surgical technique, namely Furlow palatoplasty, the findings cannot be generalized to other surgical techniques. Additionally, a small number of children were included in each group classified according to the timing of surgery. This study also has the same limitations as previous studies that relied on perceptive and subjective speech evaluations [18]. However, we attempted to mitigate this limitation by having one speech therapist consistently perform cleft palate evaluations and consulting with a faculty member specializing in cleft palate for $>10$ years.

Similarly to other researchers, we agree that an early diagnosis and intervention for SMCP significantly impact treatment outcomes. It is evident that earlier the diagnosis, as well as surgical and speech therapy interventions, the lower likelihood for secondary surgery. Moreover, less energy will be required to resolve the remaining speech problems, and a better prognosis may be expected. However, because the symptoms (functions and defects) presented by SMCP patients may vary from normal to the most severe level, we suggest that it is important to provide individualized treatment to each patient, rather than adhering to specific time periods and procedures.

\section{NOTES}

\section{Conflict of interest}

No potential conflict of interest relevant to this article was reported.

\section{Ethical approval}

The study was approved by the Institutional Review Board of 
Asan Medical Center (IRB No. 2014-1040) and performed in accordance with the principles of the Declaration of Helsinki.

\section{Author contribution}

Conceptualization: SE Jung, S Ha. Data curation: SE Jung, KS Koh, TS Oh. Formal analysis: SE Jung. Methodology: SE Jung, $S$ Ha. Project administration: SE Jung, KS Koh, TS Oh. Visualization: SE Jung. Writing - original draft: SE Jung. Writing - review \& editing: SE Jung, S Ha.

\section{ORCID}

Seung Eun Jung

Seunghee Ha

https://orcid.org/0000-0003-2512-6251

Kyung S. Koh

https://orcid.org/0000-0003-2133-3720

https://orcid.org/0000-0003-3704-4907

Tae Suk Oh

\section{REFERENCES}

1. Calnan J. Submucous cleft palate. Plast Reconstr Surg 1954; 14:1:84-5.

2. ten Dam E, van der Heijden P, Korsten-Meijer AG, et al. Age of diagnosis and evaluation of consequences of submucous cleft palate. Int J Pediatr Otorhinolaryngol 2013;77:101924.

3. Abdel-Aziz M. Treatment of submucous cleft palate by pharyngeal flap as a primary procedure. Int J Pediatr Otorhinolaryngol 2007;71:1093-7.

4. Al-Zajrawee MZ, Aljodah MA, Hassan QA. Surgical outcomes of 14 consecutive bilateral cleft lip patients treated with a modified version of the Millard and Manchester methods. Arch Plast Surg 2019;46:114-21.

5. Weatherley-White RC, Sakura CY Jr, Brenner LD, et al. Submucous cleft palate: its incidence, natural history, and indications for treatment. Plast Reconstr Surg 1972;49:297-304.

6. Garcia Velasco M, Ysunza A, Hernandez X, et al. Diagnosis and treatment of submucous cleft palate: a review of 108 cases. Cleft Palate J 1988;25:171-3.

7. Gosain AK, Conley SF, Marks S, et al. Submucous cleft palate: diagnostic methods and outcomes of surgical treatment. Plast Reconstr Surg 1996;97:1497-509.

8. Reiter R, Brosch S, Wefel H, et al. The submucous cleft palate: diagnosis and therapy. Int J Pediatr Otorhinolaryngol 2011;75:85-8.

9. Ha KM, Cleland H, Greensmith A, et al. Submucous cleft palate: an often-missed diagnosis. J Craniofac Surg 2013;24: 878-85.

10. Oji T, Sakamoto Y, Ogata H, et al. A 25-year review of cases with submucous cleft palate. Int J Pediatr Otorhinolaryngol

2013;77:1183-5.

11. McWilliams BJ. Submucous clefts of the palate: how likely are they to be symptomatic? Cleft Palate Craniofac J 1991; 28:247-9.

12. Kummer AW. Cleft palate and craniofacial anomalies: effects on speech and resonance. Toronto: Nelson Education; 2013.

13. Ruslin M, Dom L, Tajrin A, et al. Establishing cleft services in developing countries: complications of cleft lip and palate surgery in rural areas of Indonesia. Arch Plast Surg 2019;46: 511-7.

14. Kappen IFPM, Bittermann D, Janssen L, et al. Long-term follow-up study of young adults treated for unilateral complete cleft lip, alveolus, and palate by a treatment protocol including two-stage palatoplasty: speech outcomes. Arch Plast Surg 2017;44:202-9.

15. Pensler JM, Bauer BS. Levator repositioning and palatal lengthening for submucous clefts. Plast Reconstr Surg 1988; 82:765-9.

16. Abyholm FE. Submucous cleft palate. Scand J Plast Reconstr Surg 1976;10:209-12.

17. Baek RM, Kim BK, Jeong JH, et al. The effect of age at surgery and compensatory articulation on speech outcome in submucous cleft palate patients treated with double-opposing Z-plasty: a 10-year experience. J Plast Reconstr Aesthet Surg 2017;70:646-52.

18. Ettinger RE, Kung TA, Wombacher N, et al. Timing of Furlow palatoplasty for patients with submucous cleft palate. Cleft Palate Craniofac J 2018;55:430-6.

19. Smyth AG. Submucous cleft palate: outcomes after primary repair with repositioning of the levator muscle in 51 consecutive patients. Br J Oral Maxillofac Surg 2016;54:561-7.

20. Reiter R, Haase S, Brosch S. Submucous cleft palate: an often late diagnosed malformation. Laryngorhinootologie 2010;89:29-33.

21. Boyce JO, Kilpatrick N, Morgan AT. Speech and language characteristics in individuals with nonsyndromic submucous cleft palate: a systematic review. Child Care Health Dev 2018;44:818-31.

22. Ezzat AE, El-Begermy MM, Eid MI, et al. The effects of palatal lengthening on the functional and speech outcomes after submucous cleft palate repair. Otolaryngol Pol 2016;70: 41-8.

23. Ng ZY, Young SE, Por YC, et al. Results of primary repair of submucous cleft palate with Furlow palatoplasty in both syndromic and nonsyndromic children. Cleft Palate Craniofac J 2015;52:525-31.

24. Sommerlad BC, Fenn C, Harland K, et al. Submucous cleft 
palate: a grading system and review of 40 consecutive submucous cleft palate repairs. Cleft Palate Craniofac J 2004;41: 114-23.

25. Gilleard O, Sell D, Ghanem AM, et al. Submucous cleft palate: a systematic review of surgical management based on perceptual and instrumental analysis. Cleft Palate Craniofac J 2014;51:686-95.
26. Bezuhly M, Fischbach S, Klaiman P, et al. Impact of 22q deletion syndrome on speech outcomes following primary surgery for submucous cleft palate. Plast Reconstr Surg 2012;129:502e-510e.

27. Swanson JW, Mitchell BT, Cohen M, et al. The effect of Furlow palatoplasty timing on speech outcomes in submucous cleft palate. Ann Plast Surg 2017;79:156-61. 